\title{
Poli(Etileno Naftalato) - PEN: Uma Revisão do seu Histórico e as Principais Tendências de sua Aplicação Mundial.
}

\author{
Edilene de Cássia D. Nunes, J osé Augusto M. Agnelli e Roberto A. Rossi
}

Resumo: Este artigo contém uma revisão sobre o Poli(etileno naftalato) - PEN e também inclui vários aspectos relacionados com as blendas poliméricas Poli(etileno tereftalato) - PET / Poli(etileno naftalato) - PEN. O artigo é resultado de um desenvolvimento conjunto da Alcoa Alumínio S.A.- Divisão de Embalagens e do Departamento de Engenharia de Materiais - Universidade Federal de São Carlos (UFSCar), que tem como objetivo pesquisar o tema aqui abordado.

Palavras-chave: Poli(etileno naftalato) - PEN, Poli(etileno tereftalato) - PET, blendas poliméricas, embalagens.

\section{Introdução}

Atualmente há um grande interesse no desenvolvimento científico e tecnológico de materiais poliméricos destinados à área de embalagens de alimentos. Particularmente, existe uma especial atenção para as embalagens dos vários segmentos de bebidas, incluindo refrigerantes carbonatados e cervejas, além de água mineral, sucos, molhos, óleos comestíveis, defensivos agrícolas e de outros produtos diversos ${ }^{[1-24]}$.

Inicialmente, o poli(tereftalato de etileno), usualmente denominado poli(etileno tereftalato) - PET foi o principal polímero estudado e desenvolvido para as embalagens de bebidas carbonatadas ${ }^{[24]}$.

O poli(etileno tereftalato) - PET começou a ser utilizado para a produção de garrafas de refrigerantes em 1977, a partir do início da produção deste polímero com alta massa molar, através de processos de pós-polimerização em estado sólido (SSP - "Solid - State Polymerization") ${ }^{[23-26]}$.

Com relação à nomenclatura do PET, a literatura especializada é muitas vezes imprecisa. Rigorosamente, o homopolímero PET é um poliéster aromático, termoplástico, saturado, sintetizado por polimerização em etapas a partir do etileno glicol ou monoetileno glicol (EG ou MEG) e do ácido tereftálico purificado (PTA), ou com tereftalato de dimetila (DMT) substituindo o PTA. Para a fabricação de garrafas são empregados normalmente copoliésteres *, isto é, copolímeros onde o EG é o glicol principal e mais

\footnotetext{
* Copoliésteres também podem ser sintetizados usando o ácido isoftálico purificado (IPA) ou o isoftalato de dimetila (DMI) como reagente auxiliares substituindo uma pequena quantidade do PTA ou do DMT, respectivamente.
} 
um outro glicol auxiliar (utilizado em pequena quantidade) reagem com o PTA ou com o DMT. Dois exemplos de glicóis auxiliares são o cicloexano dimetanol (CHDM) e o dietileno glicol (DEG). Estes copolímeros são denominados "PET copolímero" ou PETG.

As principais diferenças entre os homopolímeros e os copolímeros são a cinética de cristalização e o comportamento térmico (transições térmicas e temperatura de distorção ao calor).

Na maioria dos trabalhos, o PET é geralmente citado sem a indicação se o polímero utilizado é um homopolímero ou um copolímero. Neste segmento de mercado, o PET, especificado industrialmente como "PET grau garrafa", normalmente possui viscosidade inerente entre $0,78 \mathrm{dL} / \mathrm{g}$ (para garrafas descartáveis) e $0,84 \mathrm{dL} / \mathrm{g}$ (para garrafas retornáveis). A viscosidade inerente (I.V. ou V.I.) é considerada baixa quando seus valores estão situados de $0,72 \mathrm{dL} / \mathrm{g}$ a $0,75 \mathrm{dL} / \mathrm{g}$, média para valores entre $0,76 \mathrm{dL} / g$ e $0,79 \mathrm{dL} / \mathrm{g}$ e alta para a faixa entre $0,80 \mathrm{dL} / g$ e $0,84 \mathrm{dL} / \mathrm{g}^{[23-28]}$.

$\mathrm{O}$ grande interesse no desenvolvimento de produtos de PET advém de suas propriedades intrínsecas, que favorecem a sua aplicação, substituindo outros materiais, tais como: metais, vidros, alguns termoplásticos de engenharia e termofixos.

Nos últimos anos surgiu um novo polímero, o poli(naftalato de etileno), freqüentemente designado como poli(etileno naftalato) - PEN, com desempenho superior ao PET, isto é, podendo ser utilizado em aplicações que exigem maiores requisitos técnicos. Entretanto, o PEN apresenta um custo elevado ${ }^{[23,25-35]}$.

\section{Propriedades e características gerais do PEN.}

O poli(etileno naftalato) ou poli(etileno-2,6naftaleno-dicarboxilato) - PEN é um poliéster aromático, termoplástico, linear e saturado, sintetizado por polimerização em etapas a partir do etileno glicol (EG) e do 2,6-éster dimetílico do ácido naftaleno dicarboxílico ou dimetil-2,6-naftaleno dicarboxilato (DM-2,6-NDC ou simplesmente NDC) ${ }^{[23,28,32,35,36]}$.

O PEN foi primeiramente sintetizado pela ICI em $1948^{[5,37]}$. Apesar da sua longa história, somente um número limitado de trabalhos têm sido publicados sobre o PEN, quando comparado com outros polímeros, como por exemplo, o PET ${ }^{[37]}$. Isto foi devido, principalmente, ao elevado custo de produção dos intermediários para a síntese do PEN. Somente em 1973 o PEN tornou-se disponível comercialmente, em peque- na escala, fornecido pela empresa Teijin Ltd - Japão, sob o nome comercial de Q-Film ${ }^{[5]}$.

A planta comercial de NDC da Amoco Chemicals, primeira unidade mundial em grande escala, concluída em 1996 com uma capacidade nominal de 27.000 toneladas/ano. A lista das empresas que estará produzindo as resinas PEN com base no NDC da Amoco é principalmente formada por: Hoechst Trevira e Akzo Nobel, na Europa; Teijin Ltd - Japão sintetizando as resinas PEN e os filmes de PEN da marca Teonex, na Ásia, e, em colaboração com a Du Pont, fornecendo PEN para as outras regiões do mundo; Eastman Chemical Company, Shell e ICI, nos Estados Unidos, sendo que a ICI produzirá os filmes Kaladex nos Estados Unidos e na Europa. No Brasil, a produtora de resinas Rhodia-Ster, que sintetiza o PET, está desenvolvendo pesquisas para sintetizar as resinas PEN ${ }^{[19,25-28,31]}$.

Para a nomenclatura do PEN, são encontradas na literatura as mesmas dificuldades observadas em relação ao PET. São disponíveis homopolímeros e copolímeros comerciais, na maioria das vezes designados apenas por PEN. Os copolímeros, onde o etileno glicol (EG) reage com diferentes proporções de NDC e PTA, normalmente apresentam duas composições predominantes indicando o nível de modificação do PEN relativo ao PET, e vice-versa: alto conteúdo de naftalato (NDC) no copolímero ["PEN modificado com $8 \%$ de tereftalato (PTA)"]; alto conteúdo de tereftalato (PTA) no copolímero ["PET modificado com $8 \%$ de naftalato (NDC)"] [19,20,30,38,39].

A inclusão do grupo naftaleno na cadeia polimérica do PEN, comparado com os anéis benzênicos do PET, aumenta a rigidez da cadeia, o que resulta em maior temperatura de transição vítrea $\left(115^{\circ} \mathrm{C}-125^{\circ} \mathrm{C}\right.$ para o PEN e $65^{\circ} \mathrm{C}-80^{\circ} \mathrm{C}$ para o PET) e maior temperatura de fusão cristalina $\left(260^{\circ} \mathrm{C}\right.$ - $270^{\circ} \mathrm{C}$ para o PEN e $240^{\circ} \mathrm{C}-255^{\circ} \mathrm{C}$ para o PET), tão bem como variação em outras propriedades, incluindo o módulo de elasticidade em tração (35\% mais elevado para o PEN), o módulo de elasticidade em flexão (50\% mais elevado para o PEN), e as resistências à tração e à fluência ${ }^{[1,12-14,17,19-23,37]}$.

Vários estudos recentes revelam que o PEN apresenta outras características de desempenho muito favorável em relação ao PET: uma melhor barreira ao oxigênio, ao dióxido de carbono e ao vapor de água (as permeabilidades ao oxigênio e ao dióxido de carbono do PEN são aproximadamente 5 vezes menores que a do PET, e a capacidade de barreira ao vapor de 
água do PEN é 3,5 vezes superior ao PET); melhor resistência química; melhor barreira aos raios ultravioleta; ciclos de moldagem mais curtos; maior temperatura de distorção térmica $\left(120^{\circ} \mathrm{C}\right.$ para o $\mathrm{PEN}$ contra $70^{\circ} \mathrm{C}$ para o PET), ${ }^{[20,26-28]}$.

Uma das maiores limitações do PET é sua baixa temperatura de distorção térmica (HDT), que como mencionado anteriormente apresenta valores em torno de $70^{\circ} \mathrm{C}$. Muitos produtos exigem pasteurização ou envase a quente, com temperaturas variando principalmente entre $75^{\circ} \mathrm{C}$ e $100^{\circ} \mathrm{C}$, e em alguns casos de pasteurização especial ou obrigatoriedade de esterilização pode-se atingir temperaturas de até $121^{\circ} \mathrm{C}$. Portanto, nestes casos, não podem ser utilizadas embalagens convencionais de PET. Somente através de processos especiais foi possível desenvolver embalagens de PET capazes de resistir ao envazamento a quente ("hot fill"), suportando temperaturas de $82^{\circ} \mathrm{C}$ a $85^{\circ} \mathrm{C}$. Para o PEN, a literatura atual consultada apresenta dados de resistência térmica, mas ainda não cita de maneira concreta uma temperatura máxima de envase a quente. São relatados desenvolvimentos bem sucedidos para temperaturas de envase entre $95^{\circ} \mathrm{C}$ e $100^{\circ} \mathrm{C}^{[20,26-28,46]}$.

Com referência à regulamentação para o emprego do PEN em contato com alimentos, na Europa e no Japão já há esta aprovação, enquanto que nos Estados Unidos a FDA (Food and Drug Administration) está avaliando o desempenho desta resina ${ }^{[28]}$.

O PEN pode ser cristalizado em dois diferentes modos cristalinos: a forma- $\alpha$ e a forma- $\beta$. Na forma- $\alpha$ as cadeias posicionam-se paralelamente ao eixo c em uma célula unitária triclínica, enquanto que na forma- $\beta$, também triclínica, cada um dos anéis de naftaleno estão rotacionados em $180^{\circ}$, dentro da célula. A literatura tem mostrado que para temperaturas de cristalização abaixo de $200^{\circ} \mathrm{C}$ preferencialmente a modificação- $\alpha$ é formada. No entanto, em temperaturas superiores a $200^{\circ} \mathrm{C}$ a modificação- $\beta$ pode ser formada se a temperatura do fundido não for muito distante do ponto de fusão ${ }^{[14]}$.

O PEN pode ser obtido no estado amorfo ou com baixa cristalinidade pelo resfriamento rápido a partir do fundido e pode ser cristalizado por orientação no estiramento, por tratamentos térmicos, ou pela combinação de ambos ${ }^{[3,7]}$. A porcentagem de cristalinidade máxima comumente observada para o PEN é de aproximadamente $45 \%{ }^{[3]}$.

As siglas APEN e APET representam as resinas PEN e PET no estado amorfo, respectivamente ${ }^{[38]}$.
O PEN não estabilizado pode ser sensível à luz, particularmente para longas exposições às radiações na região do ultravioleta (UV). Desde que ocorra absorção da radiação UV com comprimentos de onda de $382 \mu \mathrm{m}$ pode ocorrer cisão de cadeia e insolubilização por toda a amostra, acompanhada da coloração dos filmes inicialmente incolores. A radiação UV causa cisão de cadeia nas ligações carbononaftil, o que provavelmente resulta na formação de finais de cadeia naftalênicos com pouca conjugação estendida. O PEN precisa ser estabilizado com absorvedores de UV para aplicações em produtos comerciais. T. Shima et al (apud Cakmak et al ${ }^{[7]}$ ) sugerem o uso de dispersões aquosas de 2-(2-hidroxi5-metilfenil)benzotriazol ou de poli(oxietileno)nonil fenil éter para melhorar a resistência à luz do PEN.

A cinética de cristalização do PEN, a partir do polímero fundido, pode ser modificada usando-se uma variedade de agentes de nucleação, tais como, sais alcalinos de copolímeros etileno/ácidos metacrílicos e polietileno de média densidade ${ }^{[7]}$.

O PEN destaca-se por suas propriedades como: transparência (desde que a cristalinidade não ocorra ou seja mantida em níveis reduzidos); barreira ao oxigênio, ao dióxido de carbono e ao vapor de água; barreira aos raios ultravioleta, alto desempenho térmico; altas resistências mecânica, química e hidrolítica; excelente estabilidade dimensional ${ }^{[19,23,26-29,31,36,39]}$. Devido a estas características, o PEN é um polímero de grande interesse para o mercado de bebidas e alimentos com enchimento a quente, pois já foram testadas embalagens que suportam temperaturas de envase de $98^{\circ} \mathrm{C}$, enquanto que o PET quando é utilizado para produtos com envase a quente resiste a temperaturas máximas de $85^{\circ} \mathrm{C}$, em processos especiais ${ }^{[28]}$.

O principal obstáculo para o PEN é o seu custo. Uma das formas de viabilizar economicamente a aplicação do PEN é sua combinação com o PET, por meio de blendas ou pela copolimerização entre os reagentes fundamentais destes polímeros, o que certamente acarretará numa melhoria das propriedades do PET, já que uma das limitações para a utilização do PET em embalagens para bebidas carbonatadas é a sua permeabilidade gasosa. Deste modo, o desenvolvimento de misturas poliméricas PET/PEN pode minimizar esta deficiência ${ }^{[24,26,36]}$.

A relevância da incorporação de PEN ao PET, e vice-versa, é destacada por vários pesquisadores ligados à área de embalagens alimentícias. Afirma-se que a mistura polimérica PET/PEN terá para o mer- 
cado de cervejas a mesma importância do PET na área de refrigerantes ${ }^{[26,27]}$.

Uma alteração de comportamento, citada como exemplo de modificação por copolimerização, é a combinação de $2 \%$ do monômero do naftalato no "copolímero PET/PEN" * que proporciona um aumento de $90 \%$ na barreira à radiação UV. Além disto, a combinação do PEN com o PET, nas proporções adequadas, permite o uso do processo de transformação injeção-sopro e envase para temperaturas em torno de $85^{\circ} \mathrm{C}$, o que é possível para o PET somente com a utilização de técnicas especiais de injeção-sopro, que permitem a termoestabilização da garrafa ${ }^{[23,28,29]}$.

Quando se analisa o diagrama de resistência à temperatura de diferentes misturas de PET e PEN, há uma relação linear entre a mistura e a resistência à temperatura começando a $70^{\circ} \mathrm{C}$ para $100 \%$ de PET e terminando a $121^{\circ} \mathrm{C}$ para $100 \%$ de $\mathrm{PEN}^{[26]}$. A partir disto pode ser calculado que a resistência à temperatura para a mistura contendo aproximadamente $30 \%$ de PEN atinge $85^{\circ} \mathrm{C}$ e com $50 \%$ de PEN chega-se a $95^{\circ} \mathrm{C}$ como limite de temperatura para esta proporção dos polímeros constituintes da blenda polimérica ${ }^{[12,23,26,27,37,39]}$.

Existem alguns problemas quanto à obtenção das misturas de PET e PEN, as quais devem estar, se possível, ligadas quimicamente (por exemplo, através de reações de transesterificação) antes que o diagrama de temperatura da Figura 1 possa ser obedecido rigorosamente. Segundo Killestijn ${ }^{[26]}$, PET e PEN são intrinsecamente imiscíveis e formam uma mistura de duas fases. Entretanto, sob condições corretas de tempo e temperatura, ocorrerão reações de intertroca de ésteres ou transesterificação, resultando em preformas e/ou produtos transparentes.

Para produzir produtos de qualidade deve-se considerar a competição entre os pontos de fusão dos dois componentes, a competição nos aspectos reológicos, o teor de naftalato da blenda, o tamanho geral do granulado e o projeto da resina, além do equipamento de processamento, que deverá manter os dois componentes juntos por um tempo adequado ${ }^{[26]}$.

De acordo com Sherman ${ }^{[20]}$, para certas condições de processamento, com altas taxas de misturas e para tempos de residência de 1-10 minutos, os polímeros PET e PEN sofrerão uma reação química de transesterificação, produzindo um extrudado transparente. No entanto, tais tempos de residência são

\footnotetext{
* Denominação usual na literatura que aborda estes materiais ${ }^{[38,44-47]}$.
}

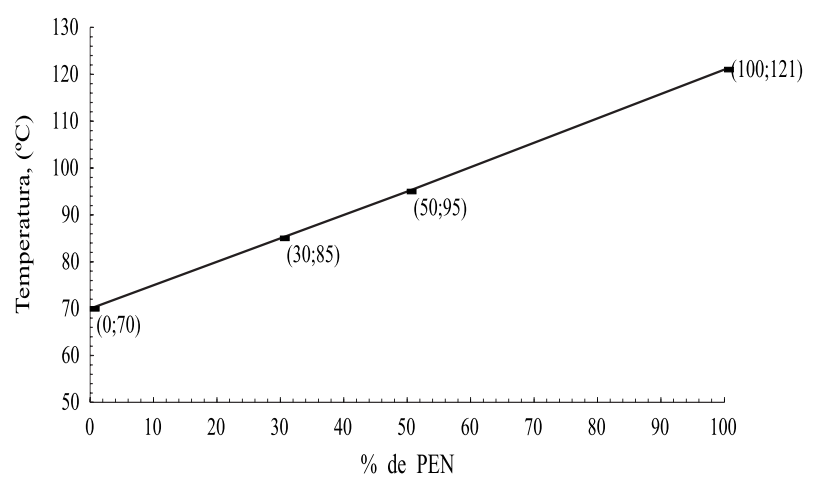

Figura 1: Diagrama de "resistência à temperatura" da blenda polimérica PET/PEN, em função do conteúdo de PEN ${ }^{[26] . ~}$

geralmente impossíveis com os equipamentos de extrusão convencionais e são indesejáveis para moldagem por injeção. Além disso, longos ciclos de mistura implicam também em perdas na viscosidade intrínseca e no aumento da produção de acetaldeído. Segundo este trabalho, há um consenso que ainda existem muitas incertezas sobre o comportamento de processamento das blendas poliméricas PET/PEN, mas pode-se concluir que o estágio de moldagem da preforma deverá ser critico para viabilizar o emprego destas blendas ${ }^{[20]}$.

Na comparação com o processamento do PET, são necessárias certas alterações nas condições de operação dos equipamentos e nos ciclos dos processos, viabilizando a sua utilização para o PEN. Por exemplo, como o ponto de fusão do PEN é mais elevado do que o do PET, o PEN requer temperaturas mais elevadas na extrusora para ser processado ${ }^{[27]}$.

Alguns pesquisadores ${ }^{[20]}$ ressaltam a obtenção de copolímeros aleatórios de PET e PEN, além das blendas. Estes copolímeros têm suportado envases a quente em temperaturas de $102^{\circ} \mathrm{C}$ contra $75-80^{\circ} \mathrm{C}$ para o PET. Além disso, recipientes reutilizáveis de PEN podem ser lavados a $85^{\circ} \mathrm{C}$ sem encolhimento, comparados com $59^{\circ} \mathrm{C}$ para o PET.

Os "copolímeros aleatórios PET/PEN" comportam-se similarmente ao PET durante estiramento e sopragem. Os copolímeros denominados de baixo nível de dimetil-2,6-naftaleno dicarboxilato (NDC), contendo menos de $15 \%$ de NDC, e os mencionados como de alto nível de NDC, contendo $85 \%$ ou mais de NDC, estão dentro da região de semicristalinidade $^{[20]}$. Tais materiais poliméricos nesta região sofrem endurecimento por deformação durante o estiramento e sopragem, o que produz um aumento nas propriedades físicas. $\mathrm{O}$ ponto exato dos limites destas regiões depende do processo utilizado e por 
quanto tempo o processo de cristalização é permitido ao copolímero ${ }^{[20,45-47]}$.

Os "copolímeros aleatórios PET/PEN" têm temperaturas de transição vítrea que são proporcionais a razão tereftalato/naftalato (razão T/N). A temperatura de fusão cristalina, no entanto, cai drasticamente a partir da temperatura de fusão dos homopolímeros, porque com a adição do comonômero, os cristais têm mais imperfeições e fundem mais facilmente. Uma explicação possível é que ocorre a formação de copolímeros não-isomorfos, onde um comonômero não substitui o outro na rede cristalina ${ }^{[45]}$.

A Figura 2 mostra as propriedades térmicas dos "copolímeros aleatórios PET/PEN".

$\mathrm{Na}$ tecnologia de moldagem por injeção-sopro (ISBM - Injection Stretch - Blow Molding) que é utilizada para fazer garrafas para bebidas carbonatadas, água mineral e uma grande quantidade de outras embalagens, o polímero deve ser forçado a ser orientado, ou endurecido sob deformação, ou cristalizado sob a tensão de estiramento. Os copolímeros de alto e baixo teor de NDC cristalizam quando estirados, e podem ser utilizados em processos ISBM. Os copolímeros amorfos contendo entre $15 \%$ e $85 \%$ de NDC não cristalizam e não podem ser utilizados nesta tecnologia ${ }^{[45]}$.

As blendas PET/PEN têm uma vantagem no processo ISBM porque oferecem a liberdade de usar toda a extensão de composições de NDC. Estas blendas têm múltiplas fases, isto é, podem ter regiões constituídas de PET ou de copolímeros com baixo teor de NDC e que deverão cristalizar, e outras regiões podem ser formadas pelo PEN ou por copolímeros com alto teor de NDC e que também deverão cristalizar,

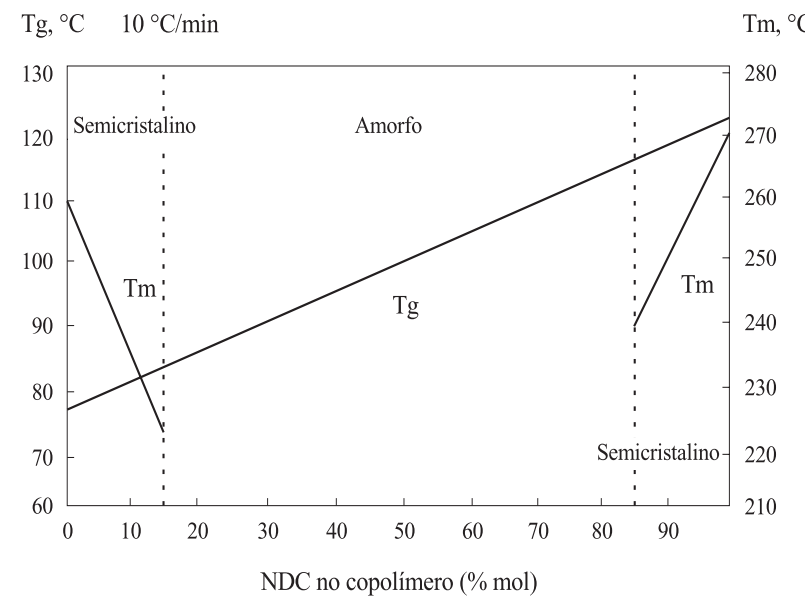

Figura 2. Dados obtídos a partir da análise por Calorimetria Diferencial de Varredura dos "copolímeros aleatórios PET/PEN" $\left(10^{\circ} \mathrm{C} / \mathrm{min}\right)^{[45]}$. enquanto a composição média da blenda pode estar entre $15 \%$ e $85 \%$ de NDC.

As blendas poliméricas podem exibir propriedades superiores àquelas que seriam esperadas pela adição das propriedades dos componentes. No caso de blendas PET/PEN, pode-se esperar mais do que as melhoras proporcionais nas propriedades de barreira. O comportamento aditivo nas misturas de dois materiais fornece uma média ponderada dos níveis de propriedades dos dois componentes: a melhoria nas propriedades é proporcional à composição. Como as blendas têm mais do que uma fase, é possível para elas apresentar comportamento de sinergismo, ou seja, propriedades melhores do que as aditivas.

Uma outra vantagem das blendas comparadas aos copolímeros, é que blendas oferecem flexibilidade para os usuários. Por exemplo, blendas pellet-pellet são misturadas um pouco antes de serem colocadas dentro do secador de resinas ${ }^{[45,46]}$.

Segundo Martin, apesar das grandes vantagens das blendas, existem alguns desafios que devem ser vencidos. Os polímeros PET e PEN são intrinsecamente imiscíveis, ou seja, um não se dissolve no outro. Assim, é necessário obter misturas dispersivas. Além disso, para se conseguir partes moldadas transparentes é muito complicado. Quando misturados eles podem se tornar translúcidos ou opacos, o que não é adequado para a etapa de reaquecimento na moldagem por sopro. Conseguir mistura e transparência, enquanto se evita degradação é difícil. A degradação das resinas é marcada por uma queda na viscosidade inerente (IV) e pela geração de acetaldeído. O desafio final é o controle da transesterificação ${ }^{[45]}$.

Quanto à transesterificação, podemos defini-la como uma reação química que ocorre entre os polímeros quando estes estão fundidos juntos. PET e PEN são ambos poliésteres e quando ocorre transesterificação pode-se formar copoliésteres. Uma pequena quantidade de transesterificação resulta, inicialmente, em copolímeros em bloco. Este copolímero em bloco tem um final de cadeia que é idêntico ao PET puro e o outro final de cadeia idêntico ao PEN puro. Quando a transesterificação continua aleatoriamente, eventualmente resulta na formação de copolímeros aleatórios. Neste caso, o arranjo de T e $\mathbf{N}$ é completamente aleatório, sendo considerado um nível de $100 \%$ de transesterificação:

Controlar a transesterificação é considerado necessário porque o efeito da transesterificação nas propriedades das blendas depende do nível de 
transesterificação conseguido. Para blendas poliméricas em geral, copolímeros em bloco são freqüentemente bons compatibilizantes o que ajuda a melhorar o desempenho da blenda, sendo que alguma transesterificação é desejada (níveis de 30-33\% para blendas). No entanto, elevada transesterificação conduz a copolímeros aleatórios que não cristalizarão e não poderão ser usados para ISBM. Alguma transesterificação pode proporcionar a obtenção de uma preforma transparente e os copolímeros em bloco atuam como compatibilizantes e ajudam a misturar a blenda mais facilmente ${ }^{[45,46]}$.

\section{Atuais mercados mundiais para o PEN e seus derivados ${ }^{[45-117]}$}

A empresa japonesa Fujiva está envasando desde o final de 1995 suas limonadas com gás em garrafas de 1,5L "de poliéster contendo PEN" "(copolímero com baixo conteúdo de naftalato (NDC) )". São utilizados materiais com baixos níveis de PEN como barreira, para proteger o produto contra a luz ultravioleta (UV). Esta barreira aos raios UV amplia a vida de prateleira do produto e protege os sucos de fruta naturais, assim como a vitamina $\mathrm{C}$ contida neles ${ }^{[58,61,63]}$.

O PEN tem aplicações como resina para embalagens devido às suas superiores propriedades térmicas, químicas, mecânicas e de barreira a gases e a luz ultravioleta. Em aplicações para embalagem, o alto desempenho faz com que o PEN seja um material ideal para o enchimento a quente, caso das marmeladas e gelatinas, e para pequenas garrafas descartáveis que necessitam ser uma barreira muito eficiente ao oxigênio e ao dióxido de carbono.

Neste caso, o NDC, precursor para a produção do PEN, foi fornecido pela Amoco Chemical Company (USA), a resina foi produzida pela Japan Unipet e a garrafa foi fabricada por Joshino do Japão ${ }^{[58,61,63]}$.

A Empresa Warner-Lambert Japan tem adotado um novo tipo de embalagem para o seu produto Listerine Cool Mint. Este é envasado em uma embalagem produzida com a blenda do PET com o PEN, onde o PEN é usado na nova aplicação para reduzir a adsorção dos ingredientes ativos contidos na solução bucal. O produto Listerine é comercializado em diferentes tamanhos que vão desde os $80 \mathrm{~mL}$ até $700 \mathrm{~mL}^{[58,61,63]}$.

As empresas envolvidas são as mesmas citadas acima, no que se refere ao NDC, ao PEN e à fabricação da embalagem.
Foi colocada no mercado uma garrafa retornável de 1,5 L, de água carbonatada Bonaqua, produzida pela Montivideo Refrescos S.A., um engarrafador do The Coca-Cola Company. Segundo a Amoco Chemical Company, este ainda é o maior teste em campo para uma garrafa PEN e constitui um passo importante no desenvolvimento do mercado de "naftalatos" para aplicações em embalagens ${ }^{[49,58,61,63]}$. Utilizando o NDC da Amoco, a resina PEN deste produto é sintetizada pela Teijin Ltd. do Japão, cuja capacidade atual é de 10.000 toneladas/ano.

A Empresa SIPA divulgou resultados iniciais sobre a fabricação e o desempenho de garrafas produzidas a partir de poliésteres à base de naftalato, com o processo integral ECS da SIPA ${ }^{[53,59]}$.

As "resinas de naftalato" utilizadas foram sintetizadas pela Hoechst Trevira, com as seguintes designações:

- Polyclear N-10 (“copolímero de baixo nível de naftalato"); VI=0,82dL/g.

- Polyclear N-90 (“copolímero de alto nível de naftalato"); VI não informada.

- Polyclear N-100 (PEN); VI=0,63dL/g.

Os produtos experimentais divulgados e avaliados pela empresa foram:

- garrafas de 2L para bebidas gasosas, fabricadas com a resina Polyclear N-10; relação média de estiramento $=12,5($ ver Tabela 1$)$.

- garrafas retornáveis de 1,5L, fabricadas com a resina Polyclear N-10; relações médias de estiramento de 10,3 e 7,1.

- garrafas de 1,5L para enchimento a quente, fabricadas com a resina Polyclear N-10; relações médias de estiramento de 13,3 e 7,8.

- garrafas âmbar de 0,33 L para cerveja, fabricadas com a resina PEN Polyclear N-100.

Entre os planos de futuros trabalhos da empresa estão a melhoria dos produtos, incluindo as otimizações dos projetos das preformas e das condições de fabricação das garrafas, e o prosseguimento com o desenvolvimento de blendas com o PEN ("blendas de naftalato").

A revista Plástico Moderno de julho/96, reproduzindo a informação publicada pela "Newsletter Plastics Brief'/julho 96, noticiou que na Áustria a Empresa Greiner und Sohne Ges, grande transformadora de resinas, está testando garrafas de PEN de 0,5L, para envase de cerveja. Dois tipos de garrafas de coloração âmbar, resistentes à radiação UV, estão sendo avaliadas. A primeira é feita de PEN homopolímero e a segunda consiste de uma blenda 
Tabela 1. Dados comparativos entre as garrafas de $2 \mathrm{~L}$, para bebidas gasosas, produzidas com o PET (AKZO DO-4300) e com o "copolímero de baixo nível de naftalato" (Polyclear N-10, Hoechst Trevira)

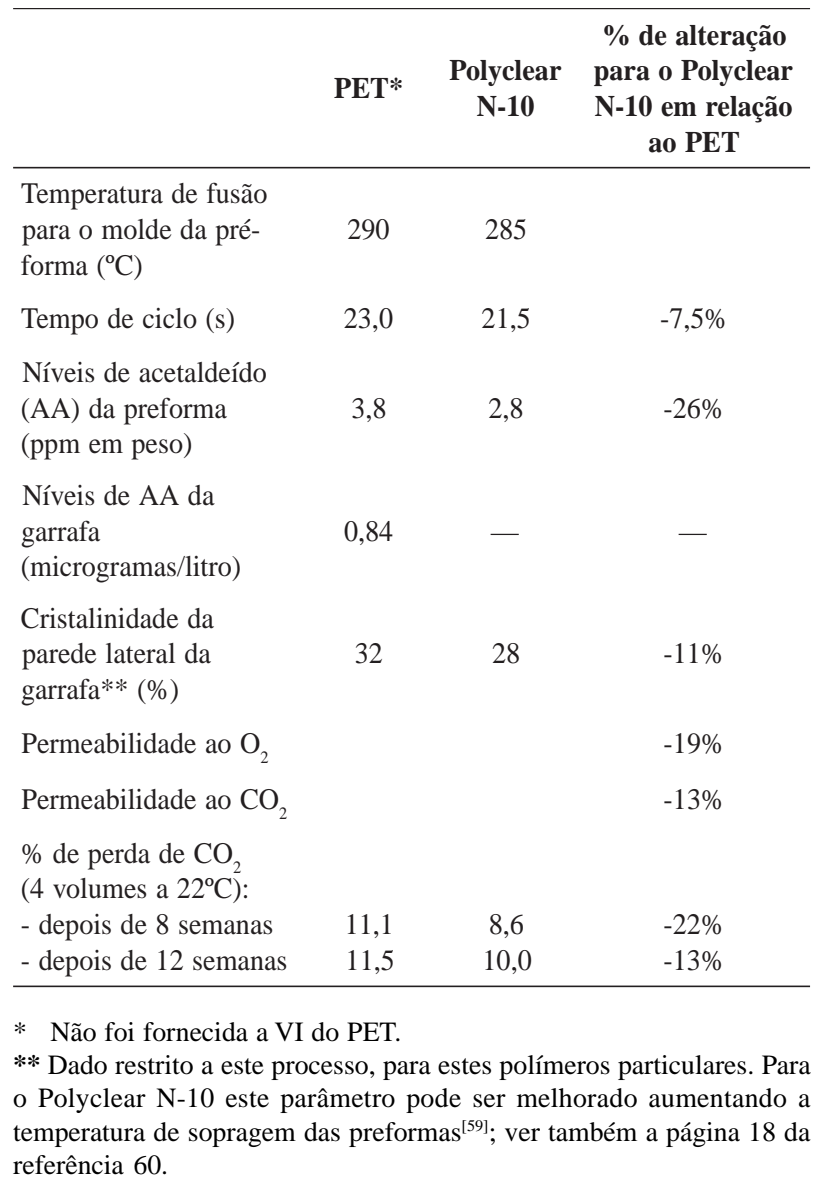

polimérica com $92 \%$ de PEN e $8 \%$ de $\operatorname{PET}^{[62]}$.

As exigências iniciais que conduziram a esta escolha são as boas propriedades em geral e a barreira ao oxigênio. Também, quanto menor é o vasilhame, maior é a relação entre a superfície da garrafa e o volume do líquido nela contido, permitindo resfriamento rápido da bebida, que não deve permanecer muito tempo no freezer. Este conjunto de exigências tornou-se um obstáculo para o PET. Além disto, a notícia conclui que para o mercado europeu, cada vez mais exigente com as normas de reciclagem dos plásticos, o PEN parece ser uma boa alternativa, por ser retornável.

A mesma notícia menciona, sem fornecer detalhes, que na Escócia começam a ser comercializados minipotes para geléias usadas na alimentação de passageiros aéreos. Assim como o PET substituiu o vidro por ser mais leve e conveniente para as empresas aéreas, o PEN tem ainda menor peso e oferece a vantagem de poder ser envasado a quente $\left(85^{\circ} \mathrm{C}\right)$.

Um trabalho publicado na revista "Tecnologia del Plástico", setembro de $1996^{[60]}$, faz uma revisão sobre o PEN e procura reforçar uma idéia de consenso da literatura técnica baseada na afirmação de que o melhor meio de fazer uso do PEN em operações de sopro é empregando as suas formas de copolímero ou através das blendas poliméricas com o PET. Além disto, a publicação menciona que muitos construtores de equipamentos para moldagem por sopro e processadores desta área têm relatado experimentos de fabricação que incluem análises detalhadas do comportamento da resina no processo e adaptações dos equipamentos para que os produtos tenham desempenho compatível com as propriedades superiores destas resinas no que se refere à resistência ao calor, resistência mecânica e barreira ao oxigênio ${ }^{[60,76,87]}$.

Com referência ao desenvolvimento de blendas poliméricas PET/PEN, considera-se que para conseguir homogeneidade da mistura devem ser ajustados os pontos de fusão das resinas e as suas viscosidades no estado fundido. Neste sentido, dois comentários foram encontrados:

- A Empresa Aoki cita que as blendas de PET com VI de 0,8 a 0,85 dL/g e PEN com VI de $0,56 \mathrm{dL} / \mathrm{g}$ são homogêneas e transparentes ${ }^{[46,60]}$.

- A Shell Chemical Company ${ }^{[38]}$ apresenta um dado empírico indicando que as resinas PET e PEN terão a mesma viscosidade de fusão quando a resina PET apresentar uma VI aproximadamente 0,25 mais alta, isto é: Viscosidade de fusão do PEN com VI = 0,60 dL/g = Viscosidade de fusão do PET com VI entre 0,80 e $0,85 \mathrm{dL} / \mathrm{g}$.

Estas relações, bem como os métodos de medir viscosidades em solução para o PEN, estão sendo muito pesquisadas ${ }^{[67]}$.

Com referência às composições dos sistemas poliméricos estudados, os principais aspectos a serem mencionados são:

- Para avaliações de peso, produtividade e custo foram comparados o PET puro, "copolímeros com $92 \%$ de PET e $8 \%$ de PEN" e blendas poliméricas PET/PEN com $8 \%$ e $15 \%$ de PEN.

- Para o processo de interesse, a faixa de composições onde é possível a utilização de blendas $\mathrm{PET} / \mathrm{PEN}$ é maior quando comparada com a região onde é viável o emprego dos "copolímeros com PEN": blendas podem ser aplicadas com a $\%$ molar de PEN entre $0 \%$ e $37 \%$ e entre $63 \%$ e $100 \%$, enquanto copolímeros são aplicáveis para “\% molar de PEN" (\% de naftalato (NDC) em relação ao PTA no copolímero) variando de $0 \%$ a $20 \%$ e de $80 \%$ a $100 \%$. 
- Para avaliações das condições de fabricação de garrafas de boca larga e das propriedades resultantes do produto são comparados os homopolímeros PET e PEN, e as blendas poliméricas PET/PEN com 10, 20 e 40\% em peso de PEN.

Nos experimentos relatados, entre outros itens importantes, é realçado que o rigoroso controle das variáveis da etapa de fabricação das preformas é fundamental para o êxito do processo. Além disto, concluiu-se que a transparência e a homogeneidade das blendas, relacionadas com o fenômeno da transesterificação ${ }^{\text {[91-96] }}$, são dependentes das temperaturas e dos tempos de residência nos equipamentos de transformação, bem como que para a obtenção de blendas poliméricas de boa qualidade devam ser evitadas as condições que provoquem elevado cisalhamento.

A empresa Hero Carters Packaging, uma divisão da Hero Drinks Group UK Ltd., está promovendo um teste de campo com garrafas sopradas com resina poliéster "PEN copolímero" HiPERTUF da Shell [54,55,61,82]. A empresa está testando dois produtos com diferentes espessuras de parede lateral: uma garrafa descartável de 59 g e uma garrafa retornável/ reutilizável de $76 \mathrm{~g}$. Ambas são projetadas para suportar enchimento a quente $\left(95^{\circ} \mathrm{C}\right)$ e vácuo causado pelo subsequente resfriamento. As garrafas foram produzidas no mesmo equipamento que produz garrafas de PET sendo as preformas injetadas numa injetora Husky e o estágio de reaquecimento/sopro é feito numa sopradora Sidel produzindo 500 garrafas $/ \mathrm{mol}$ de/hora ${ }^{[61]}$.

Uma consideração interessante relaciona-se com a resistência química do PEN e sua conseqüente aplicação em reutilizações. As garrafas do "PEN copolímero" da Carters podem ser higienizadas a $75^{\circ} \mathrm{C}$ com soda cáustica a $2 \%$, enquanto que as garrafas retornáveis/reutilizáveis de PET são limpas a $58^{\circ} \mathrm{C}$ com soda cáustica a $1,2 \%$. A eficiência e rapidez dos ciclos de limpeza são favoráveis ao "PEN copolímero"[61,83].

De modo geral, pode-se ressaltar alguns aspectos sobre o comportamento de cristalinidade do PET e do "PEN":

- O PET cristaliza mais rapidamente que o "PEN"[38].

- O "PEN", similar ao PET, permite o processo de estiramento por tensão. Este processo provoca cristalização induzida pelo estiramento, sendo esta gerada pela orientação biaxial da estrutura polimérica ${ }^{[38,60]}$.
- Comparado ao PET, o PEN suporta maiores razões de estiramento, podendo a parede lateral da garrafa ter altos níveis de cristalinidade induzida pelo estiramento ${ }^{[60]}$.

- A orientação biaxial do homopolímero PEN ocorre mais facilmente entre $140^{\circ} \mathrm{C}$ e $175^{\circ} \mathrm{C}^{[38]}$.

- Para o processo de estiramento - sopragem, que controla a cristalinidade da parede lateral da garrafa, é importante considerar os polímeros avaliados, a razão de estiramento e a temperatura empregada no processo $^{[59]}$.

\section{Principais empresas produtoras de NDC e de resinas $\mathrm{PEN}$}

As informações presentes na literatura sobre as empresas que estão produzindo NDC e produzindo/ desenvolvendo "resinas PEN" (PEN homopolímero; copolímeros com alto conteúdo de NDC e copolímeros com baixo conteúdo de NDC, indicando o nível de modificação do PEN relativo ao PET) ${ }^{[26,31,46,56,61]}$ indicam que os atuais produtores de NDC são:

- Amoco Chemicals (27.000 toneladas/ano, em 1996).

- Mitsubishi Gas Chemical.

Já os produtores ou futuros produtores de "resinas PEN" mais citados na literatura são:

- Eastman Chemical Company: 10.000 toneladas/ ano.

- Shell Chemical Co.: "resinas PEN HiPERTUF" (PEN e seus copolímeros).

- Teijin Ltd. - Japão: 10.000 toneladas/ano ("PEN Mitsui").

- Toyobo Co., Ltd. - Japão.

- Hoechst Trevira, na Europa: sem indicação de quantidade; resinas Polyclear N-10 (copolímero com baixo nível de naftalato); Polyclear N-90 (copolímero com alto nível de naftalato); Polyclear N-100 (PEN).

- Hoechst - Celanese Company, nos Estados Unidos.

- Rhodia-Ster, do Brasil: manifesta intenção de sintetizar PEN e de desenvolver blendas PET/ PEN, com $2 \%$ a $50 \%$ de PEN.

Outras empresas citadas em alguns trabalhos: Akzo Nobel; ICI; Nippon Unipet Co.; Kanebo Ltd.; Mitsubishi Chemical; Kolon.

Deve-se ressaltar que em desenvolvimentos específicos têm havido parcerias envolvendo fabrican- 
tes de matéria-prima e transformadores, como por exemplo: Shell / Amoco; Amoco / Husky / Sidel; Hero Drinks Group / Shell / Husky / Sidel; Toyobo Co. Ltd. / Aoki Technical Laboratory, Inc.

\section{Desenvolvimento de blendas poliméricas PET / PEN através do convênio de cooperação Alcoa Alumínio S.A. - Divisão de Embalagens / DEMa - UFSCar}

Em setembro de 1995 a Alcoa Alumínio S.A. Divisão de Embalagens e o DEMa - UFSCar firmaram um Convênio de Cooperação Universidade - Empresa para o desenvolvimento de blendas poliméricas PET / PEN, abrangendo a preparação, o processamento e a caracterização destas blendas poliméricas. Através do Convênio a Alcoa Alumínio S.A. - Divisão de Embalagens tem fornecido os recursos financeiros para a viabilização desta pesquisa incluindo a concessão de uma Bolsa de Doutorado e de duas Bolsas de Iniciação Científica para alunos do DEMa - UFSCar.

\section{Agradecimentos}

Os autores agradecem a Alcoa Alumínio S.A. Divisão de Embalagens, ao Conselho Nacional de Desenvolvimento Científico e Tecnológico (CNPq), ao MCT e ao MEC através do PRONEX e ao DEMa - UFSCar pelo apoio recebido.

\section{Referências Bibliográficas}

1. Cheng, S. Z. D.; Wunderlich, B.; "Glass transition and melting behavior of poly(ethylene-2,6naphthalenedicarboxylate)", Macromolecules, 21, 789-797 (1988).

2. Bark, M.; Schulze, C.; Zachmann, H.G.; "Time resolved measurements of SAXS and WAXS during crystalization and melting of polymers", Polymer Preprints, 31(2), 106-107 (1990).

3. Cheng, S. Z. D. et al.; "A study on the correlation between rigid and oriented amorphous fractions in uniaxially drawn poly(ethylene-2,6naphthalene dicarboxylate)", Polym. Bulletin, 20, 449-453 (1988).

4. Kato, T.; Kabir, G. M. A.; URYU, T.; "Solid-state $C P / M A S{ }^{13} C$-NMR studies of naphtalene-based thermotropic polyesters and model compounds",
J. of Polym. Sci.: Part A: Polym. Chem., 27, 14471465 (1989).

5. Michel, A.; Granem; Porter, R. S.; "Cold crystallization and thermal shrinkage of uniaxially drawn poly (ethylene 2,6-naphthalate) by solid-state coextrusion”, J. Polym. Sci.: Part B: Polym. Physics, 27, 2587-2603 (1989).

6. Zhou, Z.; Wu, X.; Yu, M.; "Naphthalene-ring containing thermotropic copolyester and its blends with PET", International Polymer Processing, 5(1), 37-41 (1990).

7.Cakmak, M.; Wang, Y. D.; Simhambhatla, M.; "Processing characteristics, structure development, and properties of uni and biaxially stretched poly(ethylene 2, 6 naphthalate) (PEN) films”, Polym. Eng. and Sci., 30(12), 721-733 (1990).

8. Baltá Calleja, F. J. et al.; "Influence of composition and molecular structure on the microhardness of liquid crystalline copolymers", Polymer, 32(12), 2252-2257 (1991).

9. Chen, D.; Zachmann, H. G.; "Glass transition temperature of copolyesters of PET, PEN and PHB as determined by dynamic mechanical analysis”, Polymer, 32(9), 1612-1621 (1991).

10. Ito, M.; Honda, K.; Kanamoto, T.; "Two-stage drawing of Poly(ethylene 2,6-naphtalate)", J. Appl. Polym. Sci., 46, 1013-1023 (1992).

11. Guo, M.; Zachmann, H. G.; "Intermolecular crosspolarization nuclear magnetic resonance studies of the miscibility of poly(ethylene naphthalene dicarboxylate)/poly(ethylene terephthalate) blends", Polymer, 34(12) (1993).

12. Stewart, M. E.; Cox, A. J.; Naylor, D. M.; "Reactive processing of poly(ethylene 2,6-naphthalene dicarboxylate)/poly(ethylene terephthalate) blends", Polymer, 34(19),4060-4067 (1993).

13. Huijts, R. A.; Vries, A. J.; "Mechanical Behavior of Poly(ethylene 2,6-naphthalene-dicarboxylate) (PEN) fibres near the glass-rubber transition temperature", Intern. J. Polymeric Mater., 22, 231-236 (1993).

14. Rueda, D. R. et al.; "Influence of morphology on the microhardness of poly(ethylene naphthalene2,6-dicarboxylate)", Macromol. Chem. Phys., 195, 3869-3876 (1994). 
15. Park, S. S.; Im, S. S.; Kim, D. K.; "Kinetics for the catalyzed formation of poly(ethylene 2,6naphthalate) (PEN) by various metal compounds", J. of Polym. Sci.: Part A: Polymer Chemistry, 32, 2873-2881 (1994).

16. Spies, C.; Zachmann, H. G.; "Investigation of the molecularmobility in a ternary copolyester in the liquid crystalline and isotropic states by means of deuteron N.M.R.', Polymer, 35(18), 3816-3826 (1994).

17. Abis, L.; Merlo, E.; Pó, R.; "Magic angle carbon13 NMR study of solid poly (ethylene naphthalene2,6-dicarboxylate)”, J. of Polym. Sci.: Part B: Polymer Physics, 33, 691-697 (1995).

18. Murakami, S. et al.; "A study on the structural changes during uniaxial drawing and/or heating of poly(ethylene naphthalene-2,6-dicarboxylate) films", Polymer, 36(2), 291-297 (1995).

19. "Container fabrication of naphthalate-based polyesters", Bulletin FA-18 Amoco Chemicals (april 1995).

20. Sherman, L. M.; “Get ready for PEN blow molding”, Plastics Technology (may 1995).

21. Buchner, S.; Wiswe, D.; Zachmann, H. G.; "Kinetics of crystallization and melting behaviour of poly(ethylene naphthalene-2,6-dicarboxylate)", Polymer, 30, 480-488 (1989).

22. Nakamae, K. et al.; "Elastic modulus of the crystalline regions of poly(ethylene-2,6-naphthalate)", Polymer, 34(15), 3322-3324 (1993).

23. Padula, M.; "PEN - O plástico do futuro", Informativo CETEA - Boletim do ITAL - Campinas, 6(5), 8 (1994).

24. PET - Ficha Técnica no 9 do CEMPRE (Compromisso Empresarial para Reciclagem), Rio de Janeiro (1995).

25. "PEN bottle commercialization delays appear short-term”, Modern Plastics International, 1921 (november 1995).

26. Killestijn, I. K.; "PEN/PET containers in Europe...A case study", in: Packaging Strategies'95 Conference Proceedings, separata, 10p.

27. "Will PEN be mightier than PET?", Packaging Review, 13-17 (september 1995).

28. Padula, M.; "PEN reforça propriedades do PET”, Anuário Brasileiro do Plástico, 24-25 (1995).
29. "PEN - uma opção para embalagens", Informativo ABPol, p.3 (abril/maio 1995).

30. Murphy, J.; "Amoco backs NDC potential in plastics - to the tune of $\$ 10$ million”, High Performance Plastics, p.2 (august 1995).

31. "Eastman adding PEN capacity", Polymers News, 20(8), p.247 (1995).

32. Hiraoka, K. et al.; "Synthesis and properties of polyether-polyester thermoplastic elastomer composed of poly(ethylene 2,6-naphthalate) as a hard segment", Polymer Preprints, Japan (English Edition), 42(5 11), p.E1202 (1993).

33. Nishino, T.; Gotoh, Y.; Nakamae, K.; "Elastic modulus of the crystalline regions of poly(ethylene naphthalate) and its temperature dependence", Polymer Preprints, Japan (English Edition), 42(5 11), p.E1186 (1993).

34. Murakami, S. et al.; "Study on the structural change in the drawing process of PEN poly(ethylene naphtalene - 2,6 - dicarboxylate) using the $x$ ray diffraction apparatus equipped with imaging plates", Polymer Preprints, 42(5 11), p.E1138 (1993).

35. Belcher, S.L.; "The new extrusion blow molding machinestandard PET resin”. ANTEC, 980-984 (1994).

36. Lau, O. W.; Wong, S. K.; "Permeability of naphthalene in different types of polymeric food packaging materials", Packaging Technology and Science, 8, 271-277 (1995).

37. Ülçer, Y.; Cakmak, M.; "Hierarchical structural gradients in injection moulded poly(ethylne naphthalene-2,6-dicarboxylate) parts". Polymer, 35(26), 5661-5671 (1994).

38. Callander, D.; Sisson, E.; "Resinas para packaging de alta performance basada en PEN y Naftalato", Tecnologia de PET/PEN, 1(1), 64-72 (1994).

39. "Nueva Resina PEN - Para fabricar envases resistentes al calor”, Tecnologia de PET/PEN, 1(1), 74 (1994).

40. Hage Jr., E. - "Blendas poliméricas”, Apostila Associação Brasileira de Polímeros, 136p. (1992)

41. Paul, D. R.; Barlow, J. W.; Keskkula, H.; "Polymer blends", In: Encyclopedia of Polym. Sci. and Eng'g,, Mark, H. F.; Bikales, N. M.; Overberger, 
C. G.; Menges, G. (Eds.), New York, John Wiley \& Sons, 12, 399-461 (1988).

42. Callaghan, T. A.; PAUL, D. R.; "Estimation of interaction energies by the critical molecular weight method:1. Blends with polycarbonates", J. of Polym. Sci.: Part B: Polymer Physics, 32, 1813-1845 (1994).

43. Utracki, L. A.; "Polymer alloys and blends Thermodynamics and Rheology”, Oxford University Press, New York (1990).

44. Fox, D. W.; Allen, R. B.; “Compatibility”, In: Encyclopedia of Polym. Sci. and Eng'g, Mark, H. F.; Bikales, N. M.; Overberger, C. G.; Menges, G. (Eds), John Wiley \& Sons, New York, 3, 758-775 (1988).

45. Martin, L.; "Continuing development of process technology for PET/PEN blend preforms", BEVPAK AMERICAS'95, 13-19 a 13-41 (1995).

46. Nakajima, H.; "Fabrication of naphthlatecontaining wide mouth jars", BEV-PAK AMERICAS'95, 13-81 a 13-97 (1995).

47. Sisson, E. A.; Howell, R. W.; "Shell Chemical Company HiPertuf ${ }^{\mathrm{TM}}$ resins: redefining the polyester packaging envelope for higher temperature and chemically aggressive applications", BEV-PAK AMERICAS'95, 13-1 a 13-17 (1995).

48. Andressen, E.; Zachmann, H. G.; "Studies of miscibility, transesterification and crystallization in blends of Poly(ethylene terephthalate) and Poly(ethylene-2,6naphthalene dicarboxylate)", Colloid \& Polymer Science, 272, 1352-1362 (1994).

49. "Global Teamwork Makes a Splash with PEN", Chemical Reactions Magazine, Amoco Chemicals, 12-13 (1995).

50. Agnelli, J. A. M.; “Caracterização de Polímeros por Espectroscopia no Infravermelho”. Apostila da Associação Brasileira de Polímeros, 61p. (1992)

51. ASTM D 4603-91: “Standard Test Method for Determining Inherent Viscosity of Poly(Ethylene Terephthalate) (PET)", Annual Book of ASTM Standards, Philadelphia (1995).

52. Test Method no ECC-A-AC-G-V-1-6: "Determination of Dilute Solution Viscosity of Polyesters", Eastman Chemical Company, Kingsport - Tennessee (1991).
53. "Máquinas para fabricar envases en PET, polipropileno y pruebas incluyendo PEN", Tecnologia de PET/PEN, 1(1), 49-55 (1994).

54. "Producción de envases PEN en la nueva SBO 2/3 de SIDEL”, Tecnologia de PET/PEN, 1(1), 75 (1994).

55. "SIDEL Indústria e Comércio Ltda - Todo para el soplado", Tecnologia de PET/PEN, 1(2), 10-12 (1995).

56. "Ambiente a favor pode elevar oferta de resina" Reportagem especial sobre a Brasilplast 95, Plástico Moderno, 40-47 (maio 1995).

57. Stoughton, P.; "Sistemas de secado por deshumidificación para el procesamiento com éxito de PET/PEN", Tecnologia de PET/PEN, 1(3), 29-32 (1995).

58. "El PEN sigue avanzando: Aplicaciones de importancia en Packaging”, Tecnologia de PET/ PEN, 2(4), 18 (1996).

59. Denis, G.; Masini, G.; "Actualización sobre la actividad en SIPA con poliésteres a base de naftalato". Tecnologia de PET/PEN, 2(4), 1922 (1996).

60. "PEN - Una nueva resina para botellas moldeadas por soplado”, Tecnologia del Plástico, nำ 70, 14-18 (1996).

61. "PEN writes a new chapter on container performance”, Packaging Digest, 64-65 (july 1995).

62. “Cerveja testa garrafa de PEN”. Plástico Moderno, 4-5 (julho 1996).

63. "PEN mostra aplicações". Embanews, 20, (novembro 1995).

64. Jager, J. et al;"Poly(ethylene-2,6-naphthalenedicarboxylate) Fiber for Industrial Applications”, J. Appl. Polym. Sci., 57, 14291440, (1995).

65. Ward, I. M.; Zhang, H.; "Kinetics of Hydrolytic Degradation of Poly(ethylene-2,6-dicarboxylate), Macromolecules, 28, 7622-7629 (1995).

66. Cakmak, M.; Lee, S.W.; "Molecular mechanism of neck formation in uniaxially stretched poly(ethylene naphthalate) films", Polymer, 36(21), 4039-4054 (1995). 
67. Ouchi, I. et al; "Core Electron Absorption Spectra of Polyester Films", Polymer Preprints, 42(5 11), p.E1191 (1993).

68. Peters, S. M.; Huijts, R. A.; "The relation between molecular orientation and birefringence in PET and PEN fibres", Polymer, 35(14), 3119-3121 (1994).

69. Baltá Calleja, F. J.; "Physical Aging in Poly(ethylene naphthalene-2,6-dicarboxylate) in Relation to Sorbed Water, Enthalpic Relaxation, and Mechanical Properties", J. Polym. Sci.: Part B: Polymer Physics, 33, 1653-1661 (1995).

70. Ouchi, I. et. al; "Infrared Spectra of Poly(ethylene2,6-Napthalate) and Some Related Polyesters". J. Appl. Polym. Sci., 21, 3445-3456 (1977).

71. Windle, A. H.; Lu, X.; “Crystallization of random copolymers of poly(ethylene terephthalate) and poly(ethylene naphthalene-2,6-dicarboxylate", Polymer, 36(3), 451-459 (1995).

72. Nishino, T. et al; "Temperature dependence of the elastic modulus of the crystalline regions of poly(ethylene-2,6-naphthalate)", Polymer, 36(7), 1401-1405 (1995).

73. Baltá Calleja, F. J.; "Mechanical properties and structure of glassy and semicrystalline random copolymers of poly(ethylene terephtalate) and poly(ethylene naphthalene-2,6-dicarboxilate)”, J. of Materials Sci., 27, 2161-2164 (1992).

74. Ajji, A. et al; "Amorphous orientation of poly(ethylene terephthalate) by X-ray diffraction in combination with Fourier tranform infra-red spectroscopy", Polymer, 36(21), 4023-4030 (1995).

75. Patente Européia no 051091 A2; "Polymer compositions and their blends", Becton Dickinson and Company (28/10/92).

76. Patente Européia no 0335818 A2; "Modified polyethylene naphthalate”, The Good-Year Tire \& Rubber Company (04/10/89).

77. Kim, B. S.; Jang, S. H.; "Morphology and Mechanical Properties of Liquid Crystalline Copolyester and Poly(Ethylene-2,6-Naphthalate) Blends", Polym. Eng'g and Sci., 35(6), 538-545 (1995).

78. Gohil, R. M.; Kit, K. M.; Schultz, J. M.; "Morphology and Barrier Properties of Oriented Blends of Poly(Ethylene Terephthalate) and Poly(Ethylene 2,6Naphthalate) with Poly(Ethylene-co-VinylAlcohol)". Polym. Eng'g and Sci., 35(8), 680-692 (1995).
79. Kim, B. S.; Jang, S. H.; "Rheology and Thermal Properties of Liquid Crystalline Copolyester and Poly(Ethylene-2,6-Naphthalate) Blends", Polym. Eng'g and Sci., 35(18), 1421-1431 (1995).

80. Lee, S. C.; Yoon, K. H.; "Compatibility of Poly(Ethylene-2,6-Naphthalate) and Poly(Butylene2,6-Naphthalate) Blends", Polym. Eng'g and Sci., 35(22), 1807-1810 (1995).

81. Wang, C. S.; Sun, Y. M.; "Studies on the Formation of Novel Copolyesters Containing Naphthalene and Aralkyloxy Structures", J. Appl. Polym. Sci., 58, 1189-1197 (1995).

82. Desoutter, L.; "Further Blow Molding Developments of PET/PEN Blend Bottles", BEVPAK AMERICAS'95, 13-43 a 13-61 (1995).

83. Howell, M.; "Examining Conveyor Lubricant Stress Cracking and Hot Washing Characteristics of PEN Bottles", BEV-PAK AMERICAS'95, 1363 a 13-68 (1995).

84. Koch, M.; "Preliminary Process and Container Evolution of PET/PEN Combinations", BEVPAK AMERICAS'95, 13-69 a 13-79 (1995).

85. Heinrich, M.; "Naphthalate-based Polyesters International Regulatory Update", BEV-PAK AMERICAS'95, 13-99 a 13-121 (1995).

86. Labronici, M.; "Poliéster para Garrafas - Experiência Brasileira", In: Anais do 3o Congresso de Polímeros, ABPol, p.31-34 (1995).

87. "PET Busca Agresivamente Ampliar Su Mercado", Empaque, 49, 5-7 (08-09/1995).

88. Mori, S. et al - "Structure and Physical Properties of Naphthalene Containing Polyesters I. Structure of Poly (butylene 2,6-naphthalate) and Poly(ethylene-2,6-naphtalate) as studied by Solid State NMR Spectroscopy". Polym. J., 28(2), 177181 (1996).

89. Ward, M. I.; Zhang, H.; Rankin, A.; "Determination of the end-group concentration and molecular weight of poly(ethylene naphthalene-2,6-dicarboxylate) using infra-red spectroscopy", Polymer, 37(7), 1079-1085 (1996).

90. Tate, S.; Narusawa, H.; "Thermal degradation and melt viscosity of ultra-high-molecular-weight poly(ethylene terephthalate)". Polymer, 37(9), 1583-1587 (1996). 
91. Decarli, J. R.; "Transesterificação em Blendas de Polibutileno Tereftalato (PBT)", Polímeros: Ciência e Tecnologia, 3(3), 16-20 (1993).

92. Mondragon, I.; "Control of interchange reactions of Polycarbonate/ Polyarylate blends and their influence on physical behavior", J. Appl. Polym. Sci., 32, 6191-6207 (1986).

93. Mondragon, I.; Nazabal, J.; "Influence of the level of interchange reactions on the mechanical behavior of polycarbonate / phenoxy blends", $\mathrm{J}$. of Materials Sci. Lett., 6, 698-700 (1987).

94. Mondragon, I.; Nazabal, J.; "Tensile properties of polyarylate-polycarbonate blends", Polym. Eng. and Sci., 25(3), 178-181 (1985).

95. Golovoy, A.; Cheung, M. F.; Van Oene, H.; "The phase behavior and mechanical properties of polyarylate and polycarbonate blends", Polym. Eng. and Sci., 27(20), 1642-1648 (1987).

96. Robeson, L. M.; "Phase Behavior of polyarylate blends", J. Appl. Polym. Sci., 30, 4081-4098 (1985).

97. Nitschke, C. C.; "Polyethylene terephthalate: PET, standard grades" Modern Plastics Encyclopedia Handbook, 23-26, (1994/1995).

98. Padula, M.; "Embalagem separada de PET para enchimento a quente", Boletim do CETEA-Ital, III(5), 5-6 (setembro/outubro 1991).

99. "Notícias", Anuário Brasileiro do Plástico - Editora Química e Derivados, 30-31 (1996).

100. "PET Deslancha nas Garrafas e Atrai Novos Investimentos”, Plástico Moderno, 22-28 (junho 1996).

101. Coltro, L.; "Embalagem e Ecologia: uma convivência saudável”, Engarrafador Moderno, no 45 (maio/junho 1996).

102. Jones, K. et al; "Aspects of Poly(ethylene terephthalate) Degradation for Archival Life and Environmentel Degradation", Polymer Degradation and Stability, 32, 131-159 (1991).

103. Bouneklel, M.; McNeill, I. C.; "Thermal Degradation Studies of Terephthalate Polyesters: 1. Poly(alkylene terephthalates)", Polymer Degradation and stability, 34, 187-207 (1991).

104. Allen, N. S. et al; "Physicochemical aspects of the environmental degradation of poly(ethylene terephthalate)", Polymer Degradation and Stability, 43, 229-237 (1994).
105. Vert, M. et al; "Thermal degradation of poly(ethylene terephthalate) and the estimation of volatile degradation products", Polymer Degradation and Stability, 43, 431-440 (1994).

106. Wilmes, A. et al; "Effect of some current antioxidants on the thermo-oxidative stability of poly(ethylene terephthalate)", Polymer Degradation and Stability, 44, 9-15 (1994).

107. Vince, M.; La Mantia, F. P.; "Recycling of poly(ethylenetherephthalate)", Polymer Degradation and Stability, 45, 121-125 (1994).

108. Vert, M.; "Thermal degradation of polyethylene terephthalate: study of polymer stabilization", Polymer Degradation and Stability, 49, 393-397 (1995).

109. "Coca-Cola closes the loop with multi-layer PET bottle”, Modern Plastics International, 30-31 (april 1995).

110. Bakker, M.; "Recycled content in PET bottles - a review of the options", ANTEC'94, 996-1000 (1994).

111. "Eastman Polyesters and the Environment - A success story”, Publicação MBC-104A, Eastman Chemical Company (1992).

112. Bakker, M.; "PET Recycled Content: the Focus in on Food Contact", In: Packaging Strategies'95 Conference Proceedings, separata, $15 \mathrm{p}$.

113. Oblath, R. M.; "The use of recycled polyester in packaging”, In: Anais da 8th World Conference on Packaging, 543-549 (1993).

114. "Supercycle: Impacto Mundial", Tecnologia de PET/PEN, 2(4), 9-14 (1996).

115. Wang, F. S. et al; "N.M.R. studies on the transesterificantion of polycarbonate/poly(ethylene terephthalate) blends", Polymer, 34(23), 4982-4985 (1993).

116. Paschke, E.; "Determination of Transesterification Level in Terephthalate Naphthalate Copolymers and Blends", Comunicação interna da Amoco Chemical Company (1994).

117. "O PET em maior escala" - entrevista. Embanews, 24-26 (outubro 1996).

Recebido: 22/08/97

Aprovado: 20/11/97 